\title{
Leadership \& Professional Development: Everyone Resists Change
}

\author{
Molly J Horstman, MD, MS ${ }^{1,2,3 *}$, Kyler M Godwin, PhD'1,2,3
}

${ }^{1}$ Center for Innovations in Quality, Effectiveness, and Safety, Michael E DeBakey VA Medical Center, Houston, Texas; ${ }^{2}$ VA Quality Scholars Coordinating Center, Michael E DeBakey VA Medical Center, Houston, Texas; ${ }^{3}$ Section of Health Services Research, Department of Medicine, Baylor College of Medicine, Houston, Texas.

Nothing changes without personal transformation. -W Edwards Deming, 1986

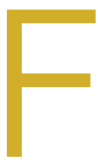

ailure is common among quality improvement projects, but also predictable. Health professionals have multiple competing priorities. Improvement projects rarely reduce an individual's workload. In our experience coaching health professionals, we have found that improvement teams often overlook two important facts: improvement requires behavior change, and everyone resists change.

Quality improvement education focuses on the development of technical skills (eg, process mapping, measure development, data analysis). Technical skills are necessary, but insufficient, to lead change. Process maps and run charts guide improvement work but alone do not motivate frontline staff to change workflows. Rather, soft skills (eg, communication, negotiation, change management, influencing others) convince frontline staff and hospital leaders that change is worth their time and effort.1,2 Successful improvement teams combine technical skills and soft skills to inspire behavior change.

We propose three practical skills that all improvement teams can adopt to inspire change:

Understand your stakeholders' needs. Early identification and engagement of stakeholders (individuals or groups who may affect or be affected by the project) is critical. Improvement teams must consider stakeholders at multiple levels in the organization, from frontline staff to executives. The easiest way to understand stakeholders is by talking to them. Often, stakeholders lack time for scheduled meetings, so teams must rely on informal conversations in hallways and elevators. The key is to understand what will motivate the stakeholder to change. Put yourself in the stakeholders' shoes: What are their needs and priorities? How might their needs and priorities motivate them to change? What potential barriers exist that prevent the stakeholder from making a change?

Tailor your message to establish a rationale for change. Build upon what was learned from stakeholders and decide how the rationale for change will be communicated. What can

*Corresponding Author: Molly J Horstman, MD, MS;

Email: molly.horstman@bcm.edu; Telephone: 713-791-1414, x10242;

Twitter: @mjhorstman.

Received: June 22, 2021; Revised: June 29, 2021; Accepted: July 7, 2021

(c) 2021 Society of Hospital Medicine DOI 10.12788/jhm.3682 you say that will influence others to see the problem as important? Recognize that the rationale is different for different stakeholders; a financial rationale may inspire hospital leaders but alienate staff who are driven by patient and staff satisfaction. Even carefully crafted messages may not resonate with stakeholders as intended. Improvement teams must monitor the impact of their message with different stakeholders. Developing a clear, concise, and compelling rationale for change is often challenging and iterative. Multiple communication channels (ie, email, newsletters, formal and informal conversations) must be employed to spread your message.

Share small and large wins. Talking with stakeholders is not a one-time event. Stakeholder interest may decrease over time. Frontline staff can become complacent, falling back into old behaviors. Priorities of hospital leadership can shift. Successful teams maintain lines of communication throughout the project to share successes and sustain stakeholder buy-in. Small and large wins matter. Project outcomes (large wins) may take months to achieve. Teams can maintain stakeholder interest by demonstrating that project processes are feasible and acceptable (small wins). Maintaining regular communication also affords teams the opportunity for early identification of organizational barriers and facilitators that may impact their project. Ongoing communication of project wins sets the stage for sustainment by embedding the change within the local culture.

The goal of any improvement project is to create sustainable change. To do this, improvement teams often need hundreds of people to change the way they work. Change is hard, but improvement teams can overcome resistance to it by strategically engaging stakeholders and thoughtfully communicating the rationale for change.

Disclosures: The authors reported no conflicts of interest.

Funding: This work is supported by the Veterans Health Administration, Office of Research Development, Leading Healthcare Improvement Training Hub grant (I50 HX002814) and the Center for Innovations in Quality, Effectiveness and Safety grant (CIN 13-413).

\section{References}

1. Myers JS, Lane-Fall MB, Perfetti $A R$, et al. Demonstrating the value of postgraduate fellowships for physicians in quality improvement and patient safety. BMJ Qual Saf. 2020;29(8):645-654. https://doi.org/10.1136/bmjqs-2019-010204

2. Rajashekara S, Naik AD, Campbell CM, et al. Using a logic model to design and evaluate a quality improvement leadership course. Acad Med. 2020;95(8):1201-1206. https://doi.org/10.1097/ACM.0000000000003191 